\title{
Serum microRNA-139-5p is downregulated in lung cancer patients with lytic bone metastasis
}

\author{
SONG XU ${ }^{1,2^{*}}$, FAN YANG $^{1,2^{*}}$, RENWANG LIU $^{1}$, XIONGFEI LI ${ }^{1}$, HAIYANG FAN $^{1}$, JINGHAO LIU $^{1}$, \\ SEN WEI ${ }^{1}$, GANG CHEN $^{1}$, JUN CHEN ${ }^{1,2}$ and YURONG DA ${ }^{3}$
${ }^{1}$ Department of Lung Cancer Surgery, ${ }^{2}$ Tianjin Key Laboratory of Lung Cancer Metastasis and Tumor Microenvironment, Lung Cancer Institute, Tianjin Medical University General Hospital, Tianjin 300052;
${ }^{3}$ Department of Immunology, School of Basic Medical Sciences, Research Center of Basic Medical Science, Tianjin Key Laboratory of Cellular and Molecular Immunology and Key Laboratory of The Educational Ministry of China, \\ Tianjin Medical University, Tianjin 300070, P.R. China
}

Received October 23, 2017; Accepted March 13, 2018

DOI: 10.3892 /or.2018.6316

\begin{abstract}
Bone remodeling can be interrupted by tumor cells which leads to an inappropriate balance of osteoblasts and osteoclasts. As the progenitors of osteoblasts, mesenchymal stem cells (MSCs) have been reported to exhibit an abnormal osteogenic differentiation potential in some cancer-related bone lesions. However, the evidence is very limited in terms of the biological alterations of MSCs in the bone metastasis of non-small cell lung cancers (NSCLC). We investigated the expression and function of miR-139-5p in MSC osteogenic differentiation in vitro in normal and NSCLC-exposed condition. Then, we compared the serum miR-139-5p in stage IV lung adenocarcinoma cancer patients with and without lytic bone metastasis. We found that MSCs exhibited a significant increase in miR-139-5p expression after exposure to osteogenic differentiation induction medium. However, Notchl, which was confirmed as a target of miR-139-5p by luciferase and western blot assays, showed a marked downregulated expression together with its pathway downstream factors during MSC osteogenesis. miR-139-5p positively regulated MSC osteogenic differentiation but this effect was abrogated significantly by Notchl knockdown. After exposure to conditions of lung cancer cells A549 and L9981, MSCs exhibited significant downregulation of miR-139-5p expression and
\end{abstract}

Correspondence to: Dr Yurong Da, Department of Immunology, School of Basic Medical Sciences, Research Center of Basic Medical Science, Tianjin Key Laboratory of Cellular and Molecular Immunology and Key Laboratory of The Educational Ministry of China, Tianjin Medical University, 22 Qixiangtai Road, Tianjin 300070, P.R. China

E-mail:dayr@tmu.edu.cn

${ }^{*}$ Contributed equally

Key words: microRNA-139-5p, mesenchymal stem cells, osteogenesis, bone metastasis, lung cancer early osteogenic marker ALP activity. Furthermore, we demonstrated that the expression of serum miR-139-5p from lung adenocarcinoma patients with lytic bone metastasis was significantly lower compared to that in patients with metastases in other organs. The potential roles of miR-139-5p as a biomarker and treatment target in monitoring and controlling bone metastasis in lung cancer patients are worthy of being further explored.

\section{Introduction}

Approximately one-third of lung cancer patients develop bone metastasis in their disease course, and these patients present with an extremely poor prognosis with a median survival of approximately 7 months (1). Bone metastasis is easy to be neglected in non-small cell lung cancer (NSCLC) patients until pain and skeletal-related events (SREs) occur. Most lung cancer bone metastatic diseases are osteolytic, and spine, ribs, pelvis and proximal long bones are commonly involved. Since the delayed demonstration of bone lesions seriously affects the survival of lung cancer patients, the identification of potentially useful and specific biomarkers is necessary for the early diagnosis of bone metastasis and follow-up.

It is well known that bone remodeling can be interrupted by tumor cells which leads to an inappropriate balance of osteoblasts and osteoclasts. Mesenchymal stem cells (MSCs) are capable of differentiating to osteoblast cells, and evidence has demonstrated that MSCs are biologically abnormal in aspects of differentiation potentials, gene expression and cytokine/chemokine secretion in the tumor microenvironment of lung cancer (2). Aberrant expression of miR-139-5p has been reported in various types of cancers (3-7). In our previous unpublished work, using a high-throughput miRNA array, we found that miR-139-5p was one of the downregulated miRNAs in MSCs from multiple myeloma patients with bone lesions as compared to those from normal donors. Therefore, we proposed that miR-139-5p may be involved in the osteogenic differentiation of MSCs as well as lung cancer bone lesions. This particular miRNA deserves further study. 


\section{Materials and methods}

Study population. The present study was conducted in accordance with the Helsinki Declaration and was approved by the Ethics Committee of Tianjin Medical University. Written informed consents were obtained from all the NSCLC patients for blood sampling and healthy volunteers for bone marrow (BM) sampling. To reduce the discrepancy, only lung adenocarcinoma patients were recruited and all of the recruited NSCLC patients with bone metastasis had only lytic bone lesions. Positron emission tomography-computed tomography (PET-CT) or emission computed tomography (ECT) was used for the diagnosis of bone metastasis together with magnetic resonance imaging (MRI) scan. No previous local or systemic treatment had been conducted before the first-time serum sample collection. Clinical data for the patients are summarized in Table I.

Primary culture of the human MSCs. The primary culture of human MSCs was performed according to our previously published protocol (8). Briefly, BM mononuclear cells were isolated by density gradient centrifugation with Ficoll-Hypaque (Nycomed; Lucron Bioproducts, De Pinte, Belgium) and seeded at a density of $1 \times 10^{6} / \mathrm{cm}^{2}$ with MesenPro medium (Invitrogen; Thermo Fisher Scientific, Inc., Waltham, MA, USA). Medium was refreshed every 3-4 days until $80-90 \%$ confluence was reached, and the cells were passaged into new flasks at 2,000 cells $/ \mathrm{cm}^{2}$. MSCs were used at passage 2 in this study. MSCs need to be characterized based on the morphology, specific immunophenotype and tri-lineage differentiation potentials.

Osteogenic differentiation induction and evaluation. To induce osteogenic differentiation in vitro, MSCs were exposed to Osteogenic Induction Medium (Lonza Group, Ltd., Basel, Switzerland) according to the manufacturer's protocol. MSCs cultured in growth medium were used as negative control. In order to examine MSC differentiation towards osteoblasts, we performed alkaline phosphatase (ALP) staining (early marker), qPCR analysis of osteoblast differentiation markers, and Alizarin Red S staining (late marker) at day 3, 7 and 14, respectively, as shown in our previous publication (8). To quantify ALP activity, we used BCIP/NBT liquid substrate and alkaline phosphatase yellow liquid substrate system for ELISA (Sigma-Aldrich; Merck KGaA, Darmstadt, Germany). Qualitative Alizarin Red S staining was used to evaluate calcium deposits. After fixation with $10 \%$ paraformaldehyde, the cells were stained with $40 \mathrm{mM}$ fresh Alizarin Red solution ( $\mathrm{pH} 4.2$ ) and destained using $10 \%$ cetylpyridinium chloride (CPC). The concentration of Alizarin Red S was determined by measuring absorbance at $562 \mathrm{~nm}$ with a multiplate reader (Thermo Labsystems, Santa Rosa, CA, USA).

Western blot analysis. The procedures involved in cell lysis, protein extraction, and immunoblotting were performed as previously described (9), using Notch1 antibody (goat polyclonal IgG; 1:500; sc-23304; Santa Cruz Biotechnology, Inc., Santa Cruz, CA, USA), followed by a horseradish peroxidase-conjugated secondary antibody (donkey anti-goat IgG; 1:2,000; cat. no. sc-2033; Santa Cruz Biotechnology, Inc.) and ECL western blotting reagents (Amersham Pharmacia Biotech, Buckinghamshire, UK).

Preparation of conditioned medium. Conditioned medium was collected according to our previous protocol (10). Briefly, conditioned medium was prepared by exposure of lung cancer cells $\left(5 \times 10^{5}\right)$ in $5 \mathrm{ml}$ serum-free RPMI-1640 medium (Lonza Group, Ltd.) for $48 \mathrm{~h}$. The culture supernatant was then harvested and centrifuged at 2,000 rpm to remove cell debris and was frozen at $-20^{\circ} \mathrm{C}$ and used within $24 \mathrm{~h}$.

Plasmid construction and dual-luciferase reporter assay. Wild-type sequences or mutant 3 ' untranslated region (3'UTR) of Notchl was constructed, carrying mutated sequences in the complementary sites for the seed region of miR-139-5p, and inserted into the pmiR-RB-REPORT ${ }^{\mathrm{TM}}$ vector (Guangzhou RiboBio Co., Ltd., Guangzhou, China). The procedure for the dual-luciferase reporter assay was previously described (8).

miR-139-5p gain- and loss-of-function analysis. Transfection of miR-139-5p inhibitor (MIN0000250; Qiagen, Leusden, The Netherlands) or miR-139-5p mimic (MSY0000250; Qiagen) was performed using Lipofectamine ${ }^{\mathrm{TM}}$ RNAiMAX reagent (Invitrogen; Thermo Fisher Scientific, Inc.) and AllStars Negative Control siRNA (1027280; Qiagen) and miScript Inhibitor Negative Control (1027271; Qiagen) were used as negative controls according to the manufacturer's protocol. Supplement of miR-139-5p mimic and inhibitor (and their controls) into osteogenic medium was refreshed every three days.

Notchl knockdown by RNA interference. The knockdown of Notch1 in MSCs was conducted by transfection with FlexiTube GeneSolution which provides four non-overlapping Notch1 RNAi duplexes (GS4851; Qiagen) together with Lipofectamine RNAiMAX reagent according to the manufacturer's protocol. AllStars Negative Control small interfering RNA (siRNA; SI03650318; Qiagen) was used as a negative control. Real-time PCR and western blot analysis were used to evaluate the knockdown efficiency.

\section{Quantitative real-time PCR ( $q P C R$ )}

$q P C R$ for miR-139-5p. For purification of microRNA from the cells, miRNA extraction and reverse transcription were performed using miReasy ${ }^{\circledR}$ Mini kit and miScript Reverse Transcription kit (Qiagen) according to the manufacturer's protocol. qPCR for miR-139-5p expression was performed using miScript SYBR-Green PCR kit (Qiagen) with iCycler Thermal Cycler system (Bio-Rad Laboratories, Nazareth, Belgium).

For purification of microRNA from human serum, blood samples were collected from lung adenocarcinoma patients. Serum was separated by centrifugation at $1,200 \mathrm{xg}$ at $4^{\circ} \mathrm{C}$ for 15 min and then frozen at $-80^{\circ} \mathrm{C}$ prior to total RNA isolation. The miRNeasy Serum/Plasma kit (Qiagen) was used to isolate RNA from the serum samples according to the manufacturer instructions. Briefly, $20 \mu 1$ total RNA sample was converted into cDNA using miScript-II-RT-kit (Qiagen). Real-time PCR was then performed with iCycler Thermal Cycler system. Reactions were performed in triplicate, and miRNA relative 
Table I. Clinical data of the NSCLC patients.

Stage IV patients without bone metastasis $(n=30)$
Stage IV patients with bone metastasis $(n=25)$

\begin{tabular}{lll}
\hline Median age (range) in years & $54(42-73)$ & $62(35-76)$ \\
Sex & & \\
Male & 14 & 12 \\
Female & 16 & 13 \\
Smoking status (\%) & 53 & 56 \\
Histology & Adenocarcinoma & Adenocarcinoma \\
Sites of metastasis & Contralateral lobe, 8 & Skeleton (vertebrae, ribs, \\
& Pleural, 12 & long bones, or pelvis), 25 \\
& Brain, 8 & \\
& Liver, 10 & \\
& Adrenal gland, 2 & \\
\hline
\end{tabular}

NSCLC, non-small cell lung cancer.

Table II. Real-time PCR primers.

\begin{tabular}{|c|c|c|c|}
\hline Gene & Primer & GenBank accession no. & Annealing temp $\left({ }^{\circ} \mathrm{C}\right)$ \\
\hline$O P N$ & $\begin{array}{l}\text { 5'-CTCCATTGACTCGAACGACTC-3' } \\
\text { 5'-CAGGTCTGCGAAACTTCTTAGAT-3' }\end{array}$ & NM_000582 & 60 \\
\hline$B S P$ & $\begin{array}{l}\text { 5'-GAATGGCCTGTGCTTTCTCAA-3' } \\
\text { 5'-TCGGATGAGTCACTACTGCCC-3' }\end{array}$ & NM_004967 & \\
\hline COLAI & $\begin{array}{l}\text { 5'-AGACGAAGACATCCCACCAATC-3' } \\
\text { 5'-AGATCACGTCATCGCACAACA -3' }\end{array}$ & NM_000088 & \\
\hline Notchl & $\begin{array}{l}\text { 5'-CTTGTGTCAACGGCGGC-3' } \\
\text { 5'-TTGGGACCGCTGAAGCC-3' }\end{array}$ & NM_017617 & \\
\hline Hesl & $\begin{array}{l}\text { 5'-AGGCTGGAGAGGCGGCTAAG-3' } \\
\text { 5'-TGGAAGGTGACACTGCGTTGG-3' }\end{array}$ & NM_005524 & \\
\hline Heyl & $\begin{array}{l}\text { 5'-GGATCACCTGAAAATGCTGCATAC-3' } \\
\text { 5'-CCGAAATCCCAAACTCCGATAG-3' }\end{array}$ & NM_001040708 & \\
\hline Runx2 & $\begin{array}{l}\text { 5'-GGAGTGGACGAGGCAAGAGTTT-3' } \\
\text { 5'-AGCTTCTGTCTGTGCCTTCTGG-3' }\end{array}$ & NM_009820 & \\
\hline$\beta$-actin & $\begin{array}{l}\text { 5'-ATGTGGCCGAGGACTTTGATT-3' } \\
\text { 5'-AGTGGGGTGGCTTTTAGGATG-3' }\end{array}$ & NM_001101 & \\
\hline
\end{tabular}

$O P N$, osteopontin; BSP, bone sialoprotein; COLA1, collagen, type I; Hes1, hairy and enhancer of split 1; Heyl, hairy/enhancer-of-split related with YRPW motif 1; Runx2, runt related transcription factor 2.

expression was calculated using a $2^{-\Delta \Delta \mathrm{Ct}}$ method. U6 was used as an endogenous control.

qPCR for osteogenic markers. mRNA extraction and reverse transcription were performed using Invitrogen ${ }^{\mathrm{TM}}$ TRIzol reagent (Thermo Fisher Scientific, Inc.), Rneasy ${ }^{\circledR}$ Mini kit (Qiagen) and Thermo Scientific Verso ${ }^{\mathrm{TM}}$ cDNA synthesis kit (Thermo Scientific, Inc.) according to the manufacturer's protocol, and miScript Reverse Transcription kit (Qiagen) according to the manufacturer's protocol. qPCR of osteogenic markers was quantified by SYBR GreenER ${ }^{\mathrm{TM}}$ qPCR for iCycler kit (Invitrogen; Thermo Fisher Scientific, Inc.) using iCycler Thermal Cycler system (8). The primer sequences used are listed in Table II.

Statistical analysis. Statistical analysis was performed by Mann-Whitney U test or one-way analysis of variance (ANOVA) followed by Tukey's post test with GraphPad 
$\mathbf{A}$

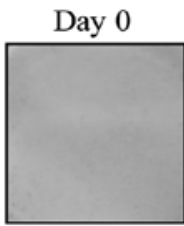

Day 2
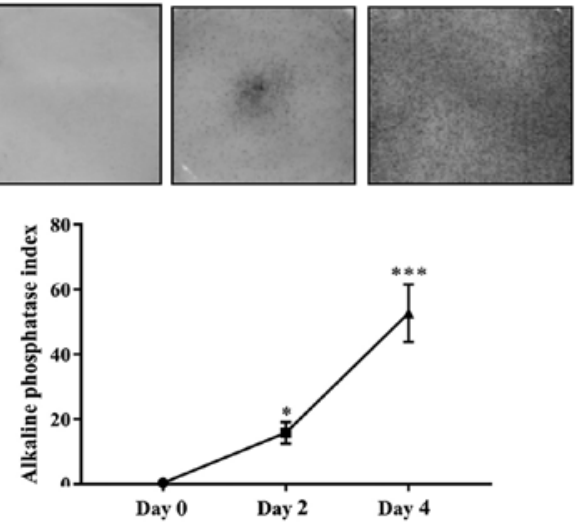

C

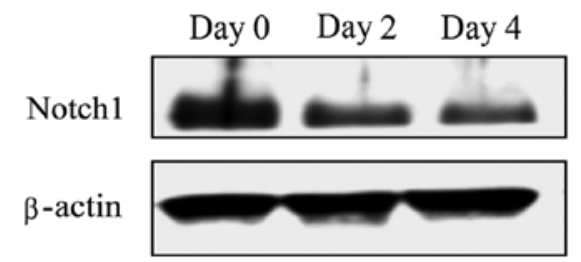

$\mathbf{E}$

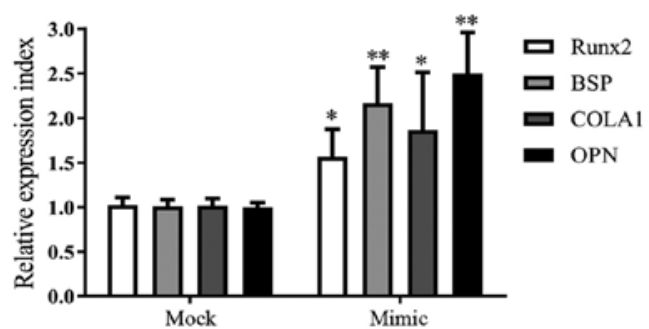

\section{G}

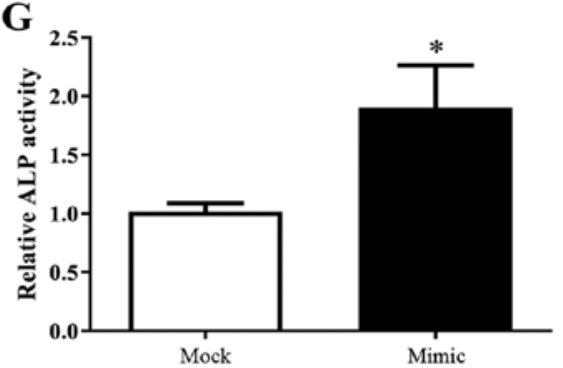

I

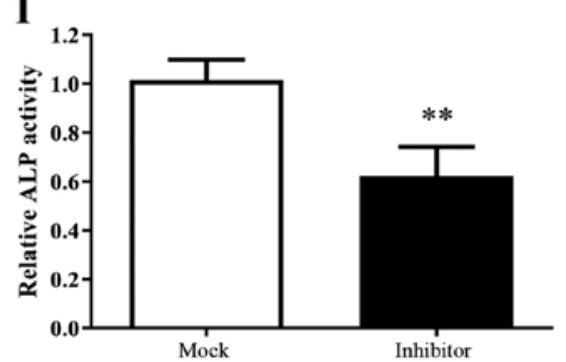

B

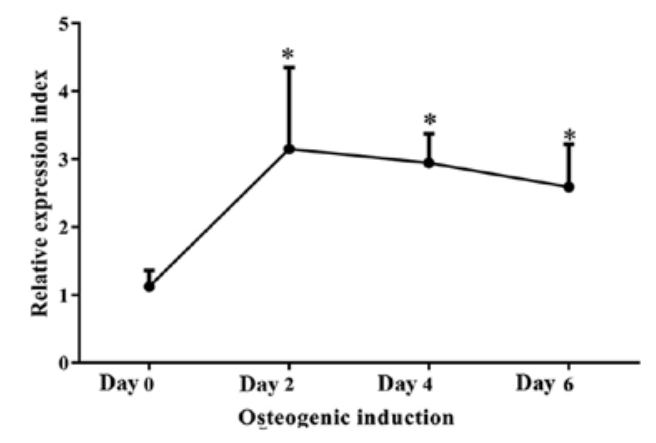

D

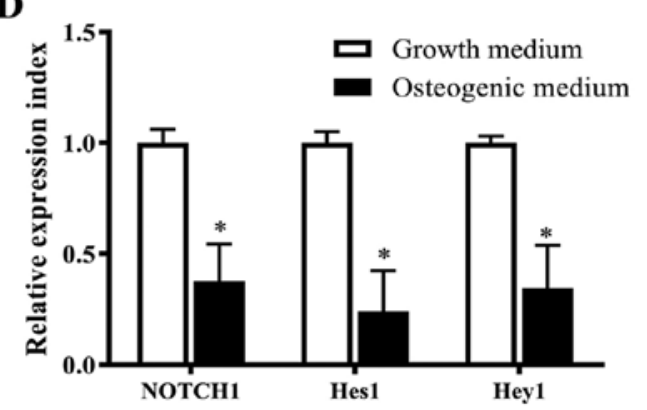

$\mathbf{F}$

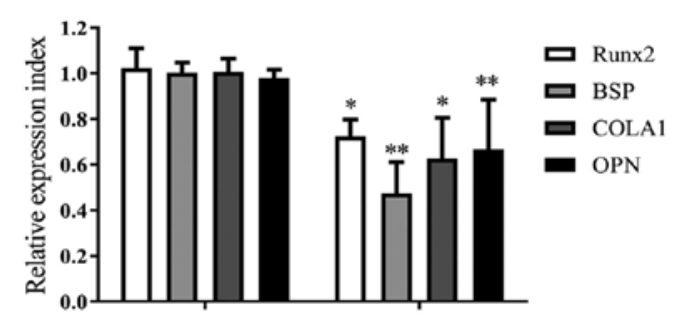

$\mathbf{H}$

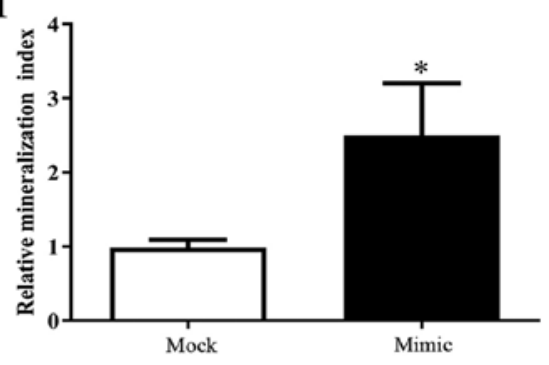

$\mathbf{J}$

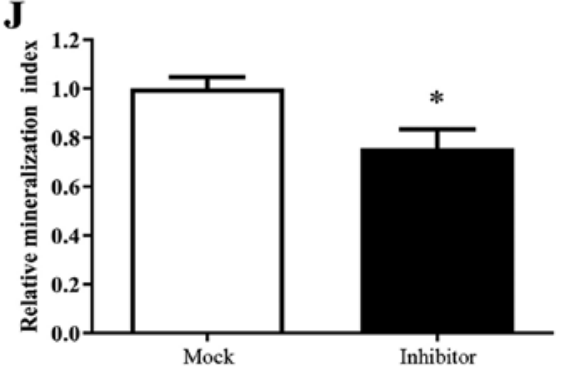

Figure 1. miR-139-5p expression is increased during MSC differentiation towards osteoblasts and positively regulates osteogenic differentiation. (A) MSCs underwent osteogenic differentiation when exposed to osteogenic induction medium in vitro as shown by ALP staining. (B) Real-time PCR test showed that miR-139-5p increased significantly during MSC osteogenic differentiation. (C) Notchl decreased significantly during MSC osteogenic differentiation by western blot analysis. (D) Notch downstream genes Hesl and Heyl decreased during MSC osteogenic differentiation as well (day 4). (E) Transfection with miR-139-5b mimic (50 nM) for $24 \mathrm{~h}$ led to upregulation of Runx2, BSP, COLA1 and OPN expression. (F) Transfection with miR-139-5b inhibitor (50 nM) for $24 \mathrm{~h}$ led to downregulation of Runx2, BSP, COLA1 and $O P N$ expression. Transfection with miR-139-5b mimic (50 nM) led to (G) upregulation of ALP activity $(72 \mathrm{~h})$ and $(\mathrm{H})$ mineralization (14 days) in MSCs. In contrast, transfection with the miR-139-5b inhibitor (50 nM) led to (I) downregulation of ALP activity (72 h) and (J) mineralization (14 days). ${ }^{*} \mathrm{P}<0.05,{ }^{* * *} \mathrm{P}<0.01,{ }^{* * * *} \mathrm{P}<0.001$. MSCs, mesenchymal stem cells; ALP, alkaline phosphatase; Run $x 2$, runt related transcription factor 2; OPN, osteopontin; BSP, bone sialoprotein; COLA1, collagen type I. 
A

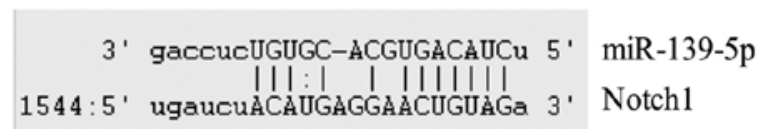

C

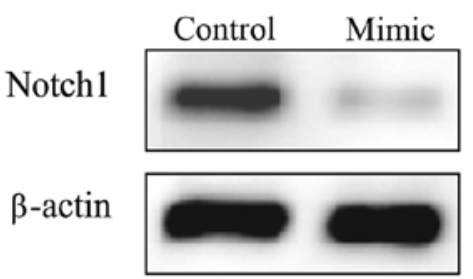

B

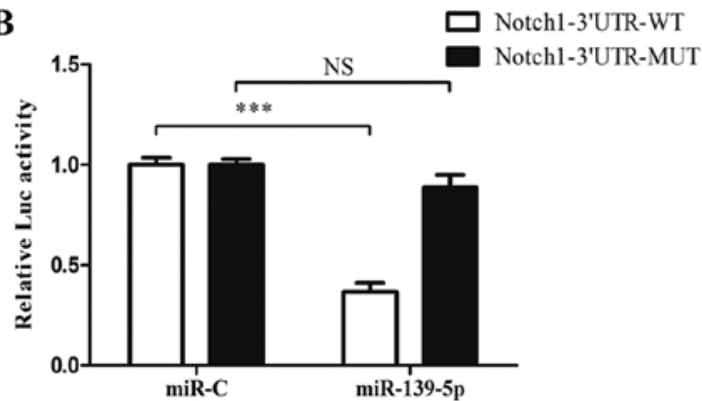

D

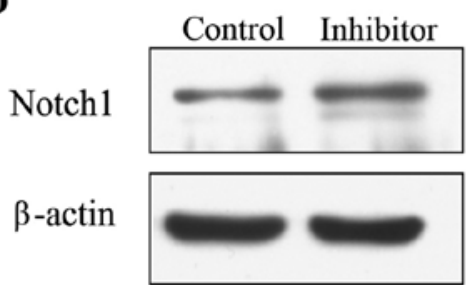

Figure 2. miR-139-5b negatively regulates Notch1 expression. (A) Bioinformatic tool predicted that the 3'UTR sequence of Notch1 is highly conserved and has binding sites for miR-139-5p (www.microrna.org). (B) miR-139-5p mimic or negative control (miR-C) (50 nM) was transfected in 293 cells for $48 \mathrm{~h}$ which were co-transfected with the luciferase reporters carrying wild-type (WT) or mutated (MUT) Notch1 3'UTR. The relative luminescence units were achieved by normalizing the luminescence of Renilla luciferase to that of firefly luciferase. Normalized data are shown as mean $\pm \mathrm{SD} ; \mathrm{n}=3$; ${ }^{* * *} \mathrm{P}<0.001 ; \mathrm{NS}$, not significant. (C and D) Western blot assay showed that transfection with miR-139-5p mimic or inhibitor (72 h) led to the downregulation or upregulation of Notch1 expression in MSCs, respectively. One representative result is shown. MSCs, mesenchymal stem cells.

Prism 7 software (GraphPad Software, Inc., La Jolla, CA, USA). P-values $<0.05$ were considered to indicate statistically significant results. All experiments were conducted at least three times.

\section{Results}

miR-139-5p and Notch1 exhibit an inverse tendency in change during MSC osteogenesis in vitro. During MSC osteogenic differentiation in vitro shown by ALP staining (Fig. 1A), we observed a considerable increase in miR-139-5p expression (Fig. 1B), and inversely a decreased expression of Notchl (Fig. 1C). As expected, this demonstrated that the expression of Notch signaling downstream genes Hesl and Heyl was widely suppressed in MSCs after culturing in osteogenic induction medium (Fig. 1D). Bioinformatic tools predicted that 3'UTR of Notchl has binding sites for miR-139-5p (Fig. 2A). Functional luciferase activity assay and western blot analysis confirmed that miR-139-5p binds directly to the predicted binding sites in the Notch1 3'UTR and negatively regulates Notchl expression (Fig. 2B-D).

miR-139-5p positively regulates the MSC osteogenic differentiation. MSCs, which were transfected with miR-139-5p mimic, exhibited a significant enhanced expression of osteogenic markers (BSP, COLA1, OPN) and Runx2 expression by real-time PCR, which is a key transcription factor for osteogenesis (Fig. 1E), as well as upregulated ALP and mineralization activities (Fig. $1 \mathrm{G}$ and $\mathrm{H}$ ). In contrast, MSCs, which were transfected with miR-139-5p inhibitor, showed a significant decreased osteogenic differentiation compared to the controls (Fig. 1F, I and J). Moreover, we observed that MSCs, which were knocked down for Notch1 expression (Fig. 3A and B), did not exhibit significant alterations in ALP activity and bone formation marker expression after treatment with miR-139-5p mimic or inhibitor (Fig. 3C-F).

Lung cancer cell-derived factors impair MSC osteogenic differentiation. After exposure to the conditioned medium of NSCLC cell lines (A549 or L9981) for 72 h, MSCs exhibited a significant downregulation in miR-139-5p expression and increased expression of Notchl expression (Fig. 4A and B). Notably, we found that ALP activity of MSCs decreased in the osteogenic induction medium mixed with NSCLC conditioned medium (Fig. 4C). However, the decreased ALP activity of MSCs induced by the NSCLC conditioned medium was recovered significantly when the MSCs were transfected with the miR-139-5p mimic (Fig. 4D).

Serum miR-139-5p is significantly lower in lung cancer patients with lytic bone metastasis. We collected blood from 55 untreated stage IV lung adenocarcinoma patients and found that serum miR-139-5p was significantly lower in lung cancer patients with bone metastasis compared to the patients with metastasis at other sites (Fig. 5A). In addition, we recruited five surgically resected lung adenocarcinoma patients (Table III) and examined their dynamic levels of serum miR-139-5p. One of five patients eventually developed an exclusive bone metastasis at the sternum. The patient (female, 37 years of age) was admitted to our department due to a persistent cough for approximately 6 months. Contrast-enhanced chest computed tomography indicated a solitary mass located in the left upper lobe which had invaded the main pulmonary artery and bronchus. A left pneumonectomy was performed with systemic lymphadenectomy, and the histology was adenocarcinoma with mediastinal lymph node metastasis of station 5 (pT2aN2M0, IIIA, AJCC 7th edition). Genetic analysis showed EGFR exon $21 \mathrm{~L} 858 \mathrm{R}(+)$. The patient received post-operative 
A

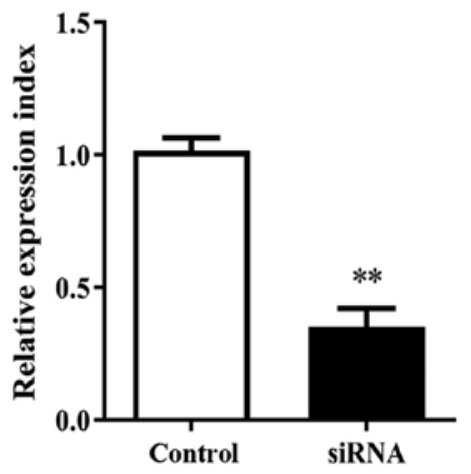

C

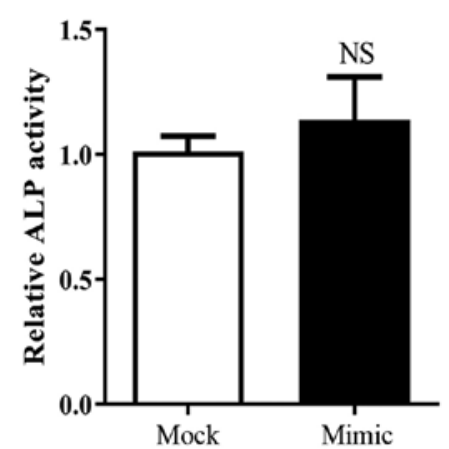

E

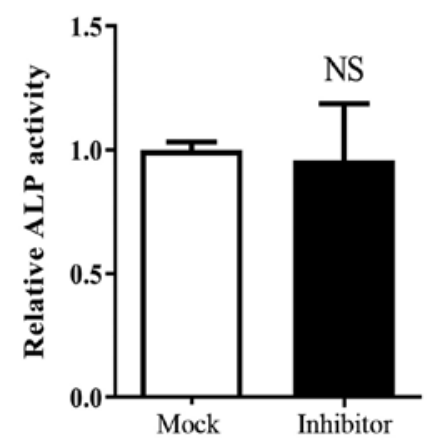

B

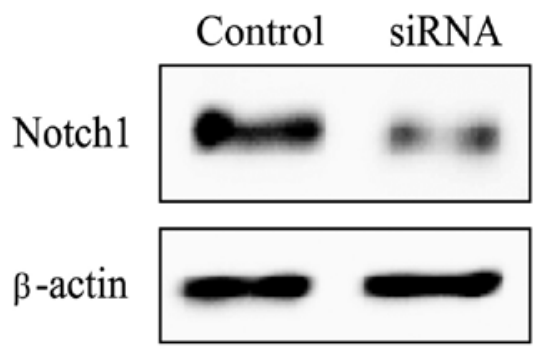

D

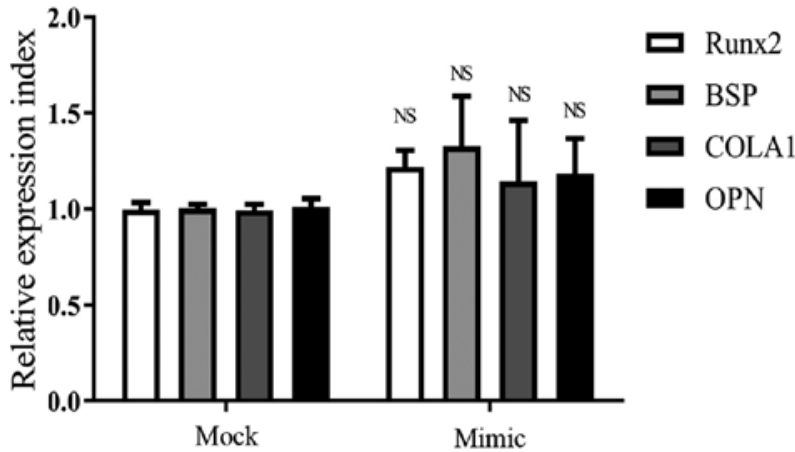

F

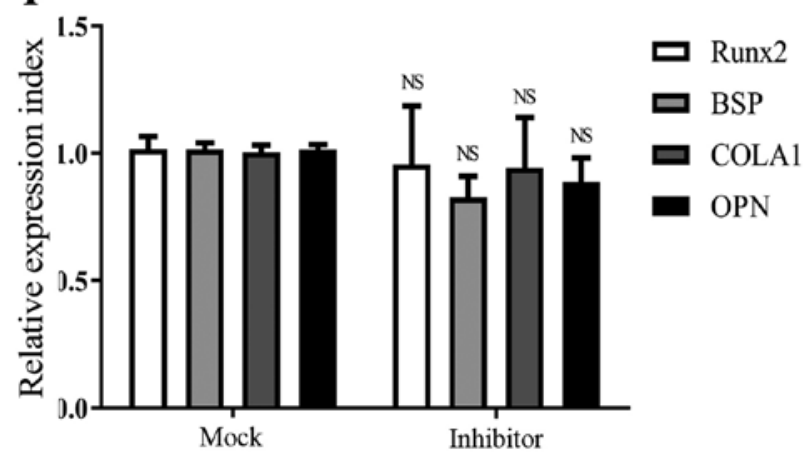

Figure 3. Knockdown of Notch1 abrogates miR-139-5p-induced MSC osteogenesis. Notch1 knockdown by siRNA transfection was confirmed by (A) real-time PCR (24 h) and (B) western blot analysis ( $72 \mathrm{~h})$. Transfection with miR-139-5b mimic (50 nM) did not lead to a significant change in (C) ALP activity (72 h) and (D) Runx2, BSP, COLA1 and $O P N$ expression ( $24 \mathrm{~h})$, in MSCs with Notch1 knockdown; Similarly, miR-139-5b inhibitor (50 nM) had no significant effect on (E) ALP activity (72 h) and (F) Runx2, BSP, COLA1 and OPN expression ( $24 \mathrm{~h})$, in MSCs with Notch1 knockdown. ${ }^{* *} \mathrm{P}<0.01$; NS, not significant. MSCs, mesenchymal stem cells; Runx2, runt related transcription factor 2; OPN, osteopontin; BSP, bone sialoprotein; COLA1, collagen type I.

Table III. Clinical features of 5 NSCLC patients who underwent dynamic serum miR-139-5p evaluation.

\begin{tabular}{|c|c|c|c|c|c|c|c|c|c|}
\hline \multirow{2}{*}{$\begin{array}{l}\text { Patient } \\
\text { no. }\end{array}$} & \multirow{2}{*}{$\begin{array}{c}\text { Age } \\
\text { (years) }\end{array}$} & \multirow[b]{2}{*}{ Sex } & \multirow[b]{2}{*}{ Surgery } & \multirow[b]{2}{*}{ Staging } & \multirow{2}{*}{$\begin{array}{l}\text { Adjuvant } \\
\text { therapy }\end{array}$} & \multicolumn{4}{|c|}{ Metastasis } \\
\hline & & & & & & Check 1 & Check 2 & Check 3 & Check 4 \\
\hline Pt1 & 71 & Male & RLL & IIA & Chemotherapy & No & No & No & $\begin{array}{l}\text { Mediastinal LN } \\
\text { (station 7) }\end{array}$ \\
\hline Pt2 & 53 & Female & RUL & IIB & Chemotherapy & No & No & No & No \\
\hline Pt3 & 61 & Male & RUL & IIIA & $\begin{array}{l}\text { Chemotherapy + } \\
\text { radiotherapy }\end{array}$ & No & No & No & Brain \\
\hline $\mathrm{Pt} 4$ & 65 & Male & LUL & IIIA & Chemotherapy & No & No & Supraclavicular LN & Supraclavicular LN \\
\hline $\mathrm{Pt} 5$ & 38 & Female & LLL & IIIA & Chemotherapy & No & Bone & Bone & Bone \\
\hline
\end{tabular}

NSCLC, non-small cell lung cancer; Pt, patient; RLL, right lower lobectomy; RUL, right upper lobectomy; LUL, left upper lobectomy; LLL, left lower lobectomy; LN, lymph nodes; Check, radiological check. 

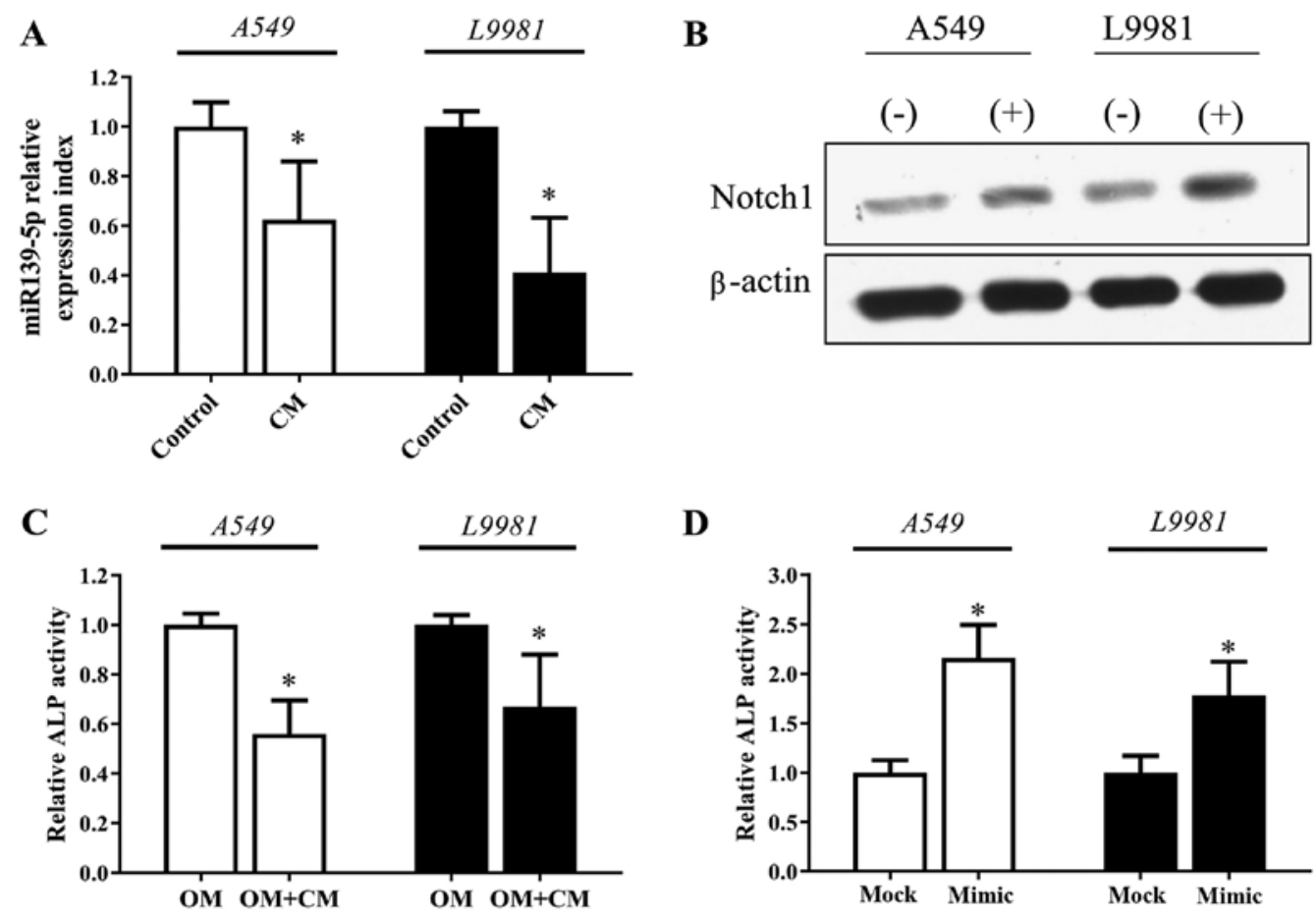

Figure 4. Lung cancer cell-derived factors impair MSC osteogenic differentiation. After exposure to the conditioned medium (CM) of NSCLC cells (A549 or L9981) for $72 \mathrm{~h}$, MSCs showed (A) significant downregulation of miR-139-5p expression and (B) increased expression of Notch1. (C) MSCs exhibited a significant decrease in ALP activity when exposed to the osteogenic induction medium mixed with NSCLC conditioned medium (OM+CM) for $72 \mathrm{~h}$. (D) Pre-transfection of miR-139-5p mimic for $24 \mathrm{~h}$ significantly recovered the decreased ALP activity of MSCs induced by the NSCLC cell-produced soluble factors. " $\mathrm{P}<0.05$. CM, conditioned medium; OM, osteogenic induction medium.

chemotherapy (pemetrex and nedaplatin, 4 cycles) and then regular imaging and serological evaluation. When bone metastasis of the sternum was detected at the 2 nd postoperative check, she started to receive icotinib daily $(125 \mathrm{mg}$ t.i.d., oral) and zoledronic acid $(0.4 \mathrm{mg}$, i.v.) each month. The patient had a significantly partial response to the treatment and the metastatic bone disease was controlled well. Since the first postoperative chemotherapy, we started to examine the serum miR-139-5p level and observed dynamic change. On the first follow-up check, we already found that miR-139-5p started to decrease but ECT and CT examinations did not report any abnormality. On the second follow-up check, the level of serum miR-139-5p continued to decrease. Meanwhile, ECT reported abnormal metabolic accumulation in the sternum and CT scan showed a lytic bone destruction in the sternum. After the patient was administered EGFR-TKI and zoledronic acid infusion, the level of serum miR-139-5p returned significantly and the ECT examination showed a less metabolic accumulation in the sternum, indicating the effectiveness of EGFR-TKI for this patient. We found that the levels of serum miR-139-5p were well correlated with the disease status and treatments for this patient (Fig. 5B). The dynamic serum miR-139-5p expression of an additional 4 patients is shown in Fig. 5C.

\section{Discussion}

Bone is among the most common sites of metastasis of lung cancer and is associated with a poor patient quality of life and survival. Most of lung cancer skeletal involvements appear lytic with apparent cortical destruction. Radiological imaging is essential in the diagnosis and monitoring of cancer-related bone lesions. However, different radiological techniques have various limitations, including lack of sensitivity for the detection of early lesions, non-specificity and high cost. If bone involvement can be predicted at its early stage, effective intervention could be implemented and may result in an improvement in survival. However, the studies of the biomarkers for the early detection of skeletal metastasis and targeted therapies to reverse bone lesions are both very limited.

Dysregulation of the bone remodeling process is characterized by an imbalance between osteoblastic and osteoclastic activity. An understanding of the mechanisms involved in bone turnover and the crosstalk between bone marrow cells and lung cancer cells contribute to the identification of novel biomarkers and therapeutic targets for bone metastasis. In the present study, we identified by gain- and loss-of-function experiments that miR-139-5p positively regulated MSC differentiation towards osteoblast cells. A previous study reported that miR-139-5p targeted Notch1 expression directly, which is in accordance with our findings (3). In addition, previous studies from our and other groups have demonstrated that the Notch pathway can maintain normal bone marrow mesenchymal progenitors in a more undifferentiated state by suppressing osteoblast differentiation (11-13). We proposed that after exposure to osteogenic induction condition, MSCs were forced to undergo epigenetic alterations, including the upregulation of miR-139-5p, which inhibited Notchl-mediated signaling activity and triggered osteogenic differentiation. This conclusion was further confirmed by the finding that miR-139-5p-induced MSC osteogenic differentiation was abrogated in Notch1-knockdown MSCs. 

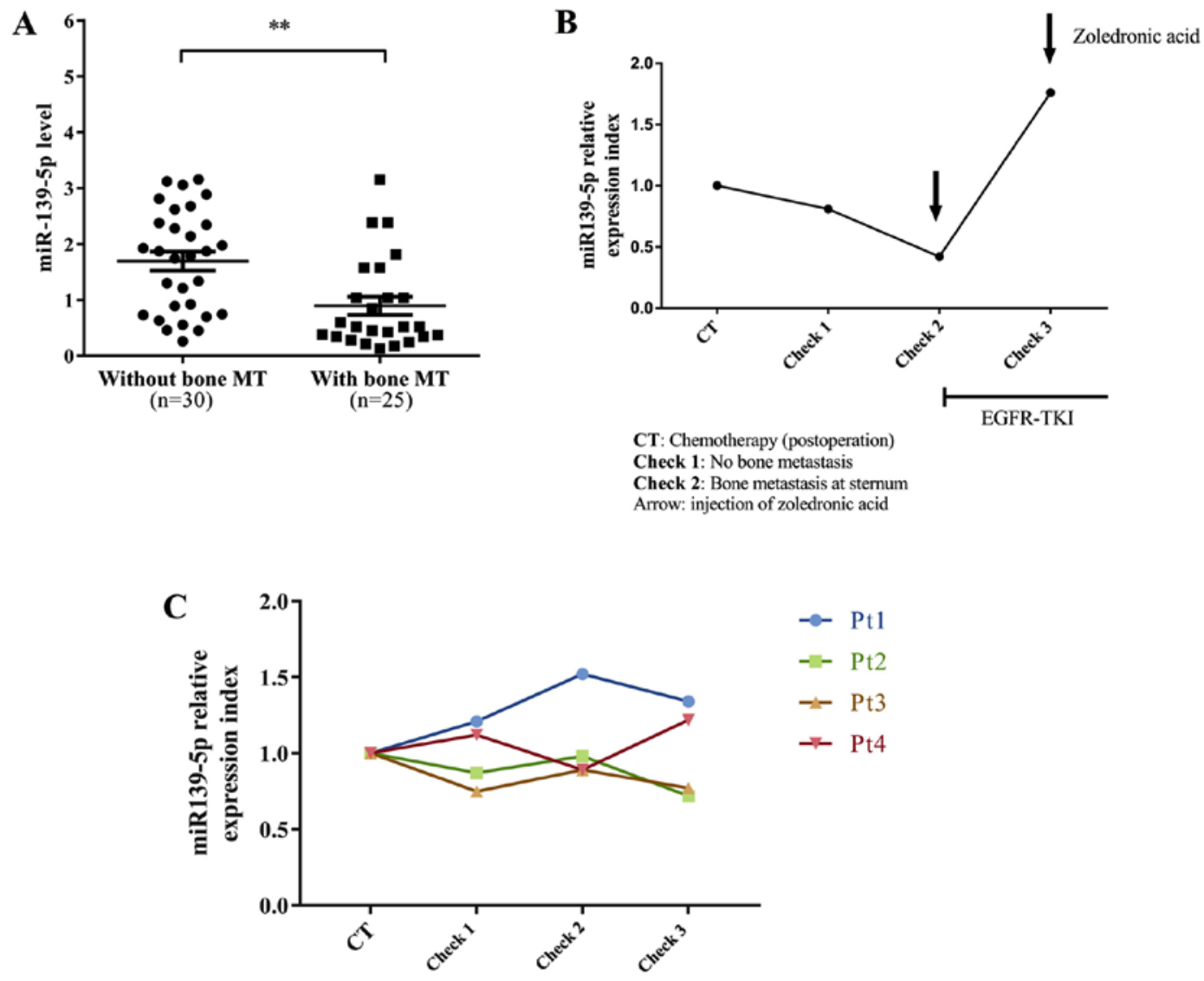

Figure 5. Serum miR-139-5p is significantly lower in lung cancer patients with lytic bone metastasis. (A) Serum miR-139-5p was significantly lower in stage IV lung cancer adenocarcinoma patients with lytic bone metastasis $(n=25)$ compared to patients with metastasis of other organs $(n=30)$. (B) One representative patient exhibited dynamic serum miR-139-5p change during disease progression and treatment response. (C) The postoperative dynamic levels of serum miR-139-5p of 4 surgically resected lung adenocarcinoma patients (Pt1-Pt4). ${ }^{* *} \mathrm{P}<0.01$. CT, chemotherapy; Check, radiological check; MT, metastasis; EGFR-TKI, epidermal growth factor receptor tyrosine-kinase inhibitor.

A number of studies have demonstrated that the crosstalk between tumor cells and MSCs through paracrine signaling could increase metastatic potential and promote epithelial-to-mesenchymal transition of tumor cells, and induce MSCs to acquire abnormal genotypes and phenotypes $(14,15)$. In our previous studies, by coculture experiments with a Transwell system, we found that MSCs favored the proliferation of lung cancer and multiple myeloma cells. In turn, tumor cells could induce epigenetic alterations and upregulate various growth factors in MSCs which promote tumor cell growth, including prointerleukin-6 (IL-6), insulin growth factor-1 and vascular endothelial growth factor $(8,16)$. In the present study, after exposure of the MSCs to the conditioned medium of lung cancer cells A549 and L9981, we observed that MSCs exhibited downregulation of miR-139-5p expression, upregulation of Notch1 expression, as well as impaired ALP activity, which is an early osteogenic marker of MSCs. Our data showed that lung cancer cell-derived soluble factors could downregulate miR-139-5p expression and impair the osteogenesis in MSCs. However, more research is necessary to identify these molecules.

There are various non-invasive liquid biomarkers used to monitor NSCLC patients with bone metastasis, including $\mathrm{N}$-telopeptide, bone-specific alkaline phosphatase, carboxy-terminal telopeptide of type I collagen, amino-terminal propeptide of type I collagen and IL-7 (17). However, none of these candidates are very reliable and widely applied in the current clinical settings due to the limited sensitivity and specificity. In the present study, we observed that serum miR-139-5p was significantly lower in lung cancer patients with lytic bone lesions compared to those without bone metastasis. Moreover, a representative case showed that the dynamic serum miR-139-5p alterations were well correlated with disease progression. The potential role of serum miR-139-5p as a non-invasive biomarker for bone metastasis of lung cancer patients warrants further investigation with larger samples.

There are some limitations to our study. Firstly, we excluded the patients with sclerotic and sclerotic/lytic mixed bone metastases in this study because we inferred that the underlying mechanisms for lytic and sclerotic bone metastatic lesions may be significantly different. The role of serum miR-139-5p in lung cancer patients with sclerotic bone metastasis needs to be further investigated. Furthermore, one previous study reported that miRNA-139-5p was markedly downregulated in NSCLC tumor tissues and cell lines, and tumor growth and invasion were inhibited by overexpression of miRNA-139-5p (4). We also observed that the level of serum miRNA-139-5p tended to be gradually downregulated during the disease progression in several NSCLC patients, but the decreased level was not as marked as that in NSCLC patients with bone metastasis. Further studies must explore an optimal cut-off value for serum miRNA-139-5p to distinguish NSCLC patients with bone metastasis from other patients at a late stage. 
Taken together, for the first time, we demonstrated that miR-139-5p plays a positive role in the regulation of MSC osteogenic differentiation which is mediated by Notchl and its signaling pathway. Importantly, the expression of serum miR-139-5p from lung adenocarcinoma patients with lytic bone metastasis was significantly lower compared to the patients with metastasis at other sites. Upregulation of miR-139-5p expression may contribute to control lytic bone disease in lung cancer. Therefore, the potential roles of miR-139-5p as a biomarker and treatment target in monitoring and controlling bone metastasis in lung cancer patients are worthy of further investigation.

\section{Acknowledgements}

Not applicable.

\section{Funding}

The present study was funded by the National Natural Science Foundation of China (nos. 81772464 and 81773207), the Science and Technology Support Key Program of Tianjin (no. 17YFZCSY00840), the Tianjin Key Project of Natural Science Foundation (no. 17JCZDJC36200), the Natural Science Foundation of Tianjin (no. 16JCYBJC24600) and the Tianjin Educational Committee Foundation (no. 20120117).

\section{Availability of data and materials}

The datasets used during the present study are available from the corresponding author upon reasonable request.

\section{Authors' contributions}

SX and YRD conceived and designed the study. SX, FY, RWL, XFL, HYF and JHL performed the experiments. SX and FY wrote the manuscript. SW, GC, JC and YRD reviewed and edited the manuscript. All authors read and approved the manuscript and agree to be accountable for all aspects of the research in ensuring that the accuracy or integrity of any part of the work are appropriately investigated and resolved.

\section{Ethics approval and consent to participate}

The present study was conducted in accordance with the Helsinki Declaration and was approved by the Ethics Committee of Tianjin Medical University. Written informed consents were obtained from all the NSCLC patients for blood sampling and healthy volunteers for bone marrow sampling.

\section{Consent for publication}

Not applicable.

\section{Competing interests}

The authors declare that they have no competing interests.

\section{References}

1. Coleman RE: Clinical features of metastatic bone disease and risk of skeletal morbidity. Clin Cancer Res 12: 6243s-6249s, 2006.

2. Liu R, Wei S, Chen J and Xu S: Mesenchymal stem cells in lung cancer tumor microenvironment: Their biological properties, influence on tumor growth and therapeutic implications. Cancer Lett 353: 145-152, 2014.

3. Zhang L, Dong Y, Zhu N, Tsoi H, Zhao Z, Wu CW, Wang K, Zheng S, Ng SS, Chan FK, et al: microRNA-139-5p exerts tumor suppressor function by targeting NOTCH1 in colorectal cancer. Mol Cancer 13: 124, 2014.

4. Xu W, Hang M, Yuan CY, Wu FL, Chen SB and Xue K: MicroRNA-139-5p inhibits cell proliferation and invasion by targeting insulin-like growth factor 1 receptor in human non-small cell lung cancer. Int J Clin Exp Pathol 8: 3864-3870, 2015.

5. Dai S, Wang X, Li X and Cao Y: MicroRNA-139-5p acts as a tumor suppressor by targeting ELTD1 and regulating cell cycle in glioblastoma multiforme. Biochem Biophys Res Commun 467: 204-210, 2015.

6. Yonemori M, Seki N, Yoshino H, Matsushita R, Miyamoto K, Nakagawa $M$ and Enokida $H$ : Dual tumor-suppressors miR-139-5p and miR-139-3p targeting matrix metalloprotease 11 in bladder cancer. Cancer Sci 107: 1233-1242, 2016.

7. Dai H, Gallagher D, Schmitt S, Pessetto ZY, Fan F, Godwin AK and Tawfik O: Role of miR-139 as a surrogate marker for tumor aggression in breast cancer. Hum Pathol 61: 68-77, 2017.

8. Xu S, Cecilia Santini G, De Veirman K, Vande Broek I, Leleu X, De Becker A, Van Camp B, Vanderkerken K and Van Riet I: Upregulation of miR-135b is involved in the impaired osteogenic differentiation of mesenchymal stem cells derived from multiple myeloma patients. PLoS One 8: e79752, 2013.

9. Menu E, Kooijman R, Van Valckenborgh E, Asosingh K, Bakkus M, Van Camp B and Vanderkerken K: Specific roles for the PI3K and the MEK-ERK pathway in IGF-1-stimulated chemotaxis, VEGF secretion and proliferation of multiple myeloma cells: Study in the 5T33MM model. Br J Cancer 90: 1076-1083, 2004.

10. Xu S, Menu E, De Becker A, Van Camp B, Vanderkerken K and Van Riet I: Bone marrow-derived mesenchymal stromal cells are attracted by multiple myeloma cell-produced chemokine CCL25 and favor myeloma cell growth in vitro and in vivo. Stem Cells 30: 266-279, 2012

11. Zanotti S, Smerdel-Ramoya A, Stadmeyer L, Durant D, Radtke F and Canalis E: Notch inhibits osteoblast differentiation and causes osteopenia. Endocrinology 149: 3890-3899, 2008.

12. Hilton MJ, Tu X, Wu X, Bai S, Zhao H, Kobayashi T, Kronenberg HM, Teitelbaum SL, Ross FP, Kopan R and Long F: Notch signaling maintains bone marrow mesenchymal progenitors by suppressing osteoblast differentiation. Nat Med 14: 306-314, 2008

13. Xu S, Evans H, Buckle C, De Veirman K, Hu J, Xu D, Menu E, De Becker A, Vande Broek I, Leleu X, et al: Impaired osteogenic differentiation of mesenchymal stem cells derived from multiple myeloma patients is associated with a blockade in the deactivation of the Notch signaling pathway. Leukemia 26: 2546-2549, 2012.

14. Gunn WG, Conley A, Deininger L, Olson SD, Prockop DJ and Gregory CA: A crosstalk between myeloma cells and marrow stromal cells stimulates production of DKK1 and interleukin-6: A potential role in the development of lytic bone disease and tumor progression in multiple myeloma. Stem Cells 24: 986-991, 2006.

15. Yu PF, Huang Y, Xu CL, Lin LY, Han YY, Sun WH, Hu GH, Rabson AB, Wang Y and Shi YF: Downregulation of CXCL12 in mesenchymal stromal cells by TGF $\beta$ promotes breast cancer metastasis. Oncogene 36: 840-849, 2017.

16. Li M, Wu Y, Liu R, Guo L, Xu T, Chen J and Xu S: Investigational study of mesenchymal stem cells on lung cancer cell proliferation and invasion. Zhongguo Fei Ai Za Zhi 18: 674-679, 2015 (In Chinese).

17. Roato I: Bone metastases: When and how lung cancer interacts with bone. World J Clin Oncol 5: 149-155, 2014. 